论 文

\title{
新元古代-寒武纪与二叠-三叠纪转折时期生物和 地质事件及其环境背景之比较
}

\author{
沈树忠", 朱茂炎, 王向东, 李国祥, 曹长群, 张华 \\ 现代古生物学和地层学国家重点实验室, 中国科学院南京地质古生物研究所, 南京 210008 \\ *E-mail: szshen@nigpas.ac.cn
}

收稿日期: 2010-03-04; 接受日期: 2010-07-28

国家重点基础研究发展计划项目(编号: 2006CB860400, 2011CB808905)、中国科学院方向性项目(编号: KZCX2-YW-Q08-4)、国家自然科 学基金(批准号: 40810029002, 40921091)和中国科学院国际伙伴计划项目资助

\begin{abstract}
摘要 新元古代-寒武纪和二叠-三叠纪转折时期由于分别发生了最为壮观的生物辐射事 件(寒武纪生物大爆发)和最具灾难性的生物灭绝事件 (二叠纪末生物大灭绝), 被认为是地球 生命演化史中两个最为关键的转折时段. 然而, 最新的一些研究表明新元古代-寒武纪和二 叠-三叠纪转折时期的地质与生物演化历史具有一定的相似性, 许多新元古代-寒武纪之交 发生的重大生物与地质事件在古生代-中生代之交重复发生, 这些事件包括与地球深部地幔 柱活动有关的地表大陆重组、大规模冰川的形成和消融、与地表和大气环境变化密切相关 的 $\mathrm{C}, \mathrm{Sr}, \mathrm{S}$ 同位素波动、碳酸盐岩的异常沉积以及生物的多次辐射和大规模灭绝等. 这些事 件的重复发生表明地球深部活动是地表环境发生急剧变化的主要动因, 而地表环境的改变 导致生物多样性发生剧变. 进一步深入比较表明, 这两个转折时期虽然发生重复性的重大 生物、地质和地球化学异常事件, 但具体型式、幅度和过程有明显差别. 新元古代的 Rodinia 大陆在雪球事件以前就已经开始解体, 而石炭纪至二叠纪的 Pangea 大陆在大冰期以后才开 始裂解. 生物更替的水平比较表明新元古代-寒武纪之交主要反映在埃迪卡拉生物群的集群 灭绝和大量新的生物门类占领新的生态空间, 是一级新的生态系统的形成过程; 而二叠纪 末生物大灭绝虽然造成大量生物灭绝、造礁作用和成煤作用停止, 但主要反映在纲和目一级 生物类群的灭绝与新生, 大灭绝后, 残存的生物在早三叠世晚期开始重新占领生态空间, 复 苏时间也远远短于寒武纪早期的小壳动物发展阶段, 是一种生态系统结构组成的重大变化. 与生物大规模更替相伴生的两个关键转折时期的碳同位素等均发生了强烈波动, 但波动的 幅度和频率有所不同, 说明当时的大气和海水介质条件和地质背景均存在明显差别. 超级 联合古陆、大规模的冰期形成和消融、 $\mathrm{C}, \mathrm{Sr}$ 和 $\mathrm{S}$ 等同位素的强烈波动以及生物多次大灭绝 和辐射等重复发生在新元古代-寒武纪与二叠-三叠纪转折时期给我们带来了新的思考, 从 地球系统整体观出发, 开展从地球深部到地表过程的交叉合作研究对认识地球系统各个圈 层的相互作用具有重要意义.
\end{abstract}

关键词

新元古代-寒武纪 二叠-三叠纪

转折时期 生物事件 地质事件 环境背景 
最近二十年以来, 地球系统科学的研究在时空 两个不同尺度上不断拓展. 地球生命历史的研究已 经进入了一个传统古生物学与地学和其他学科加速 交叉、结合的时代. 这种日趋广泛的地球系统演化的 综合研究令人信服地表明, 地球历史中所发生的各 种生物事件与当时地球本身各种自然条件的演变有 着单一学科研究所意想不到的关联性, 地球生命演 化过程中所发生的许多生物事件, 其实伴随有多种 复杂的地质事件背景 ${ }^{[1,2]}$, 人们开始探讨地球生命漫 长的起源过程与当时地球水圈、大气圈和岩石圈演变 之间的必然联系 ${ }^{[3]}$. 古生代所发生的三次生物大灭绝 与当时地球大气的 $\mathrm{O}_{2}$ 和 $\mathrm{CO}_{2}$ 含量变化、海平面变化、 海水缺氧环境和火山活动存在的联系 ${ }^{[4 ~ 7]}$ 已经得到越 来越多地质证据的支持. 全球性的系统观念正在成为 认识地球生命历史中一系列复杂的演变事件的思潮, 多学科交叉的研究手段成为必不可少的技术途径.

大量研究表明地球生命演化的进程并不是一帆 风顺的，而是经历了许许多多相对比较短暂的“波 折” 以及相对漫长的“稳定”时期, 生命在某些历史时 段发生大规模爆发(biological explosion)或者大灭绝 (mass extinction) 等剧烈的演变, 这些时段被称为关 键转折时期(critical transitions). 其中, 因发生了地质 历史时期最为壮观的生物辐射事件(寒武纪生物大爆 发)和最具灾难性的生物大灭绝事件 (二叠纪末生物 大灭绝), 新元古代-寒武纪和二叠-三叠纪转折时期 已经普遍被认为是地球生命演化史中两个影响最大 的关键时段 ${ }^{[8 \sim 10]}$. 令人惊奇的是, 最近的一些研究表 明, 新元古代-寒武纪与二叠-三叠纪转折时期的地质 与生物演化历史具有比较明显的相似性, 许多新元 古代-寒武纪之交发生的重大生物与地质事件同时在 二叠-三叠纪之交重复发生(图 1), 因此, 二叠-三叠纪 转折时期的研究被认为可能是揭开新元古代-寒武纪 之交所发生的一系列重大事件之谜的钥匙 ${ }^{[10,17 ~ 20]}$. 本文就地球生命历史中这两个最为关键的时段所发 生的生物与地质事件进行比较分析, 从而为探索生 物重大演化事件与地球环境变化的协同关系提供参 考.

\section{1 新元古代-寒武纪与二叠-三叠纪转折时期 的生物事件}

自 25 亿年前的元古代初期至 5.2 亿年前的寒武
纪早期, 地球生命历史完成了从没有可见生命的荒 芜状态向生物繁茂的现代蓝色星球状态的转变. 在 这一时期内, 从真核生物开始出现到多细胞动植物 的起源经历了一个漫长的过程. 然而, 大约 6.3 5.2 亿年之间的新元古代晚期至寒武纪早期是地球生命 历史中的一个加速演化阶段 ${ }^{[21,22]}$. 大约在 6.2 亿年前, 多细胞藻类开始繁盛. 嗣后, 在 5.8 亿年前后, 地球 生命进入了一个全新的动物宙时代 ${ }^{[23]}$, 微型的后生 动物胚胎、两侧对称动物, 大型复杂的埃迪卡拉生物 群以及寒武纪早期大量具矿化外壳和骨骼化生物等 相继出现 ${ }^{[23 ~ 28]}$. 根据已有的研究资料, 新元古代至寒 武纪初生物大爆发前, 至少有 5 次生物新生事件: 1)雪 球事件后 “大型带刺疑源类”和大型多细胞藻类在伊 迪卡拉纪早期的辐射；2) 后生动物胚胎和埃迪卡拉 生物群在埃迪卡拉纪中期的大量出现；3）弱骨骼化 管状动物、遗迹化石和生物扰动构造在埃迪卡拉纪晚 期的出现；4）小壳动物化石在寒武纪初的大爆发；5) 以澄江动物群为代表的几乎所有已知的动物门类的 快速起源(寒武纪生命大爆发) ${ }^{[24,29,30]}$. 与此同时, 早 期出现的许多生物类群经过短暂的演化后大部分并 没有延续下来, 其中一部分在新元古代末期灭绝, 包 括“大型带刺疑源类”在埃迪卡拉纪中期的消亡 ${ }^{[31,32]}$ 和埃迪卡拉生物群在新元古代与寒武纪之交的整体 灭绝等. 新元古代末可能发生了地史时期中的第一 次生物大灭绝, 即埃迪卡拉生物群大灭绝 ${ }^{[23,27,33 ~ 35]}$, 只是在这之前由于宏观生物种类较少, 大灭绝规模 无法与显生宙的数以万计的物种灭绝在数量上相比 较, 从而常常被演化生物学家们所忽视. 然而新元古 代与寒武纪之交的生物大规模更替可以根据宏观后 生动物分异度和多样性面貌的巨大差异、从遗迹化石 所反映的生物行为复杂化的转变和生物扰动强度的 增强等容易识别 ${ }^{[36,37]}$ (图 1). 埃迪卡拉纪生物集群灭 绝之后, 以生物骨骼化为特征的梅树村小壳化石大 量出现揭开了寒武纪大爆发的序幕. 到距今 5.2 亿年 的寒武纪早期, 动物的辐射达到了顶峰, 辐射在门级 分类单元上表现最为显著, 不仅几乎所有主要的现 生后生动物门业已出现 ${ }^{[26]}$, 而且还发育许多短暂延 续的、亲缘关系不明的干群, 发生了被科学界誉为当 今自然科学十大谜题之一的“寒武纪生物大爆 发”,[21,38 40] (图 2). 在中国, 围绕着新元古代-寒武纪 这一转折时段内的生物群, 包括蓝田生物群、瓮安生 物群、庙河生物群、高家山生物群、梅树村动物群、 







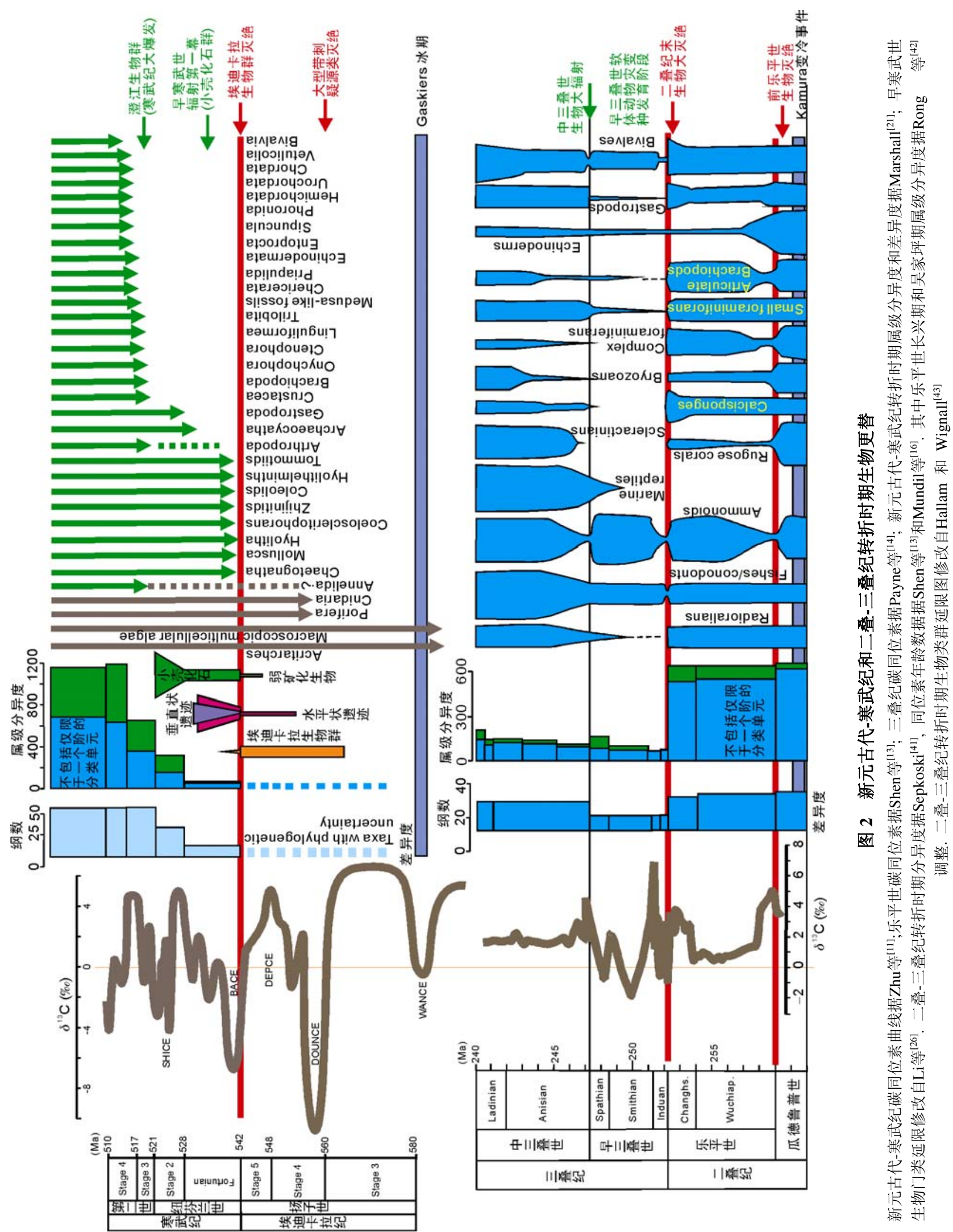


澄江生物群、关山生物群和凯里生物群等的研究大大 丰富了新元古代-寒武纪过渡期生物多样性, 从而证 实了新元古代-寒武纪转折时期地球生命发生了多次 重大更替.

寒武纪大爆发后, 经过奥陶纪生物大辐射, 地球 生命进入了一个多样性繁荣而稳定的演化阶段 ${ }^{[8]}$. 在 整个古生代的演化历史中, 虽然发生了奥陶纪末和 泥盆纪晚期两次生物灭绝事件, 但这两次事件对整 个生物多样性结构并没有产生根本性的影响, 尤其 是在高一级生物分类单元上影响很小. 然而, 二叠纪 晚期地球生态系统发生了又一次重大变革, 完成了 从古生代动物群向现代动物群的转变. 根据已有研 究, 石炭纪-乌拉尔世(早二叠世)的生物稳定繁荣, 全 球陆地上发育了最大规模的森林, 海洋中各门类生 物(例如: 珊瑚类、簉类、腕足类、牙形类等)和陆生 脊椎动物两栖类等丰富多彩, 进入乌拉尔世晚期, 生 物多样性有一个稳定增长时期. 瓜德鲁普世(中二叠 世)开始, 生物多样性开始进入一个强烈波动的时期. 瓜德鲁普世末全球范围内发生了一次显著的生物更 替事件, 称为前乐平世事件 ${ }^{[44]}$ 或瓜德鲁普世末期事 件 ${ }^{[45 ~ 50]}$. 这次生物灭绝事件明显具有生物类群选择 性 ${ }^{[48]}$. 一方面, 簿类、珊瑚类等遭受重创 ${ }^{[49,51]}$, 瓜德 鲁普世以后这两个类群全球生物多样性明显下降, 从此再也没有恢复到乐平世以前的水平. 另一方面, 腕足类、腹足类、双壳类等在属一级水平上发生明显 变化, 牙形类也发生属级水平的变革, 以Jinogondolella为代表的类群被以Clarkina为代表的类群所取 代, 但从瓜德鲁普世至乐平世属级以上水平的总体 分异度并没有发生实质性的变化 ${ }^{[45,50]}$, 只是一些瓜 德鲁普世的一些特征属消失, 从而说明这是一次在 生物群落结构水平上(相当于Droser等 ${ }^{[52]}$ 的最低级生 态水平)发生的一次变化, 而且多数生物的消失与当 时大规模生物栖息地的减少有密切关系 ${ }^{[48]}$. 二叠纪 早、中期海洋生物相当繁盛的北美大陆、冈瓦纳大陆、 欧洲大陆和西伯利亚板块的大部等均由于海水退出 成为陆地, 大量生活于这些地区的海洋生物从此销 声匿迹 ${ }^{[48]}$. 前乐平世生物灭绝事件以后, 以华南为中 心的特提斯暖池成为乐平世(晚二叠世)海洋生物多 样性的中心, 生物多样性和生物群地方性特色急剧 升高使得该地区成为一个非常容易遭到重创的生物 多样性热点地区(biodiversity hotspot). 不久以后, 二 叠纪末发生了显生宙最大规模的生物灭绝事件, 这
次生物大灭绝事件点断了整个生物界演化的历史进 程 ${ }^{[8]}$, 几乎对所有门类生物造成重大影响(图 2), 导致 海洋中约 $95 \%$ 的物种和陆地上约 $75 \%$ 的生物彻底消 失 ${ }^{[33,54]}$. 多个重要类群包括簿类、四射珊瑚类, 三叶 虫类、古生代非常繁盛的长身贝类、石燕贝类等遭受 灭顶之灾; 无脊椎动物界从以被动摄食的底栖生物 时代转入以主动摄食的游泳型生物为主导的时代; 脊椎动物从以两栖类为主导地位的时代转入了以恐 龙占主导地位的爬形动物时代; 植物界从蕨类植物 时代转入裸子植物时代. 从此, 结束了整个古生代的 历史. 二叠纪末生物大灭绝后, 生物经历了一个长约 5 百万年的生物萧条期 ${ }^{[5]}$, 其复苏时间长度为显生宙 5 次生物大灭绝之最, 当时海洋中微生物和低等生物 非常繁盛 ${ }^{[56 ~ 59]}$, 新元古代晚期和寒武纪初反映大量 微生物活动的微生物岩在大灭绝以后的三叠纪初再 次广泛出现, 海洋世界一度回到与新元古代末期相 似的原始时代, 直至早三叠世末和中三叠世初, 生物 才得于再一次辐射 ${ }^{[14,55]}$, 地球生命的演化从此进入 了一个新的爬行动物和裸子植物时代, 这次现代生 物的大规模辐射几乎可以与寒武纪生物大爆发相媲 美(图 1,2).

\section{2 新元古代-寒武纪与二叠-三叠纪转折时期 的地质事件}

最近的许多研究表明, 地球早期多细胞生物的 出现、辐射和消亡以及其后的寒武纪大爆发与当时剧 烈的环境变化有密切关系. 这些变化包括大陆重组、 海洋和大气化学组成、气候变化等 ${ }^{[21]}$. 新元古代与寒 武纪之交发生的重大地质事件(图 1)主要包括: 1) 在 中元古-新元古代之交, 全球大陆在低纬度集合形成 一个Rodinia联合大陆 ${ }^{[60,61]}$, 这一大陆自新元古代早 期开始分裂, 在Rodinia联合大陆解体以后, 大约在 6 5.2 亿年期间在南半球地体重组形成了一个影响整 个古生代的冈瓦纳大陆. 因此, 多细胞生物在新元古 代早期的出现、后生动物的分异以及随后的寒武纪生 物大爆发等生物事件与当时地体的重组在时空上相 吻合 ${ }^{[62]}$;2) 联合大陆开始分裂一方面增加了大陆边 缘海的范围, 增强了大陆风化作用, 促进了生物的原 始生产率, 加大了全球范围内有机碳埋藏, 这一过程 导致大气 $\mathrm{CO}_{2}$ 的急剧下降, 使得地球表面温度逐渐降 低导致冰盖的迅速扩展, 最后使地球陆地和海洋为 
冰雪所覆盖, 从而导致雪球事件的发生 ${ }^{[61,63,64]}$. 雪球 事件使得生命的存在面临一次严峻的挑战, 不过在 寒冷冰期环境下保存的生命也成为酝酿着一个全新 的生物世界的摇篮 ${ }^{[65]}$. 雪球事件以后, 新元古代中、 晚期大量动物休眠卵 ${ }^{[66]}$ 、蓝田生物群 ${ }^{[67]}$ 、大型带刺 疑源类等的出现表明这一时期地球已经进入了一个 新的时代, 同时也伴随有短暂的小规模冰期事件 ${ }^{[68]}$, 冰期事件的发生与随后的㸗安生物群 ${ }^{[23]}$ 和大量后生 动物胚胎化石的出现可能有密切的关系 ${ }^{[63]}$.

令人惊奇的是, 类似于Rodinia联合大陆的解体、 冈瓦纳大陆的重组、大规模冰期以及火山喷发等事件 在古生代-中生代之交同样存在. 从石炭纪中晚期到 二叠纪早期是Pangea联合古陆的聚合阶段 ${ }^{[69,70]}$, 这个 联合大陆大约在瓜德鲁普世早期开始转入裂解阶段, 伴随着Pangea联合大陆的解体发生了大规模火山喷 发 ${ }^{[7,71,72]}$. 华南的峨眉山玄武岩、乐平统的几十层火 山灰沉积和西伯利亚二叠-三叠纪之交的大火成岩省 等的形成都与Pangea联合大陆的解体有关 ${ }^{[73]}$. 从晚 石炭世至乌拉尔世是显生宙以来最大规模的冰川发 育时期 ${ }^{[7476]}$, 冰川几乎覆盖了整个冈瓦纳大陆, 使 得冈瓦纳大陆当时发育了非常独特的Eurydesma冷水 动物群和大规模冰川沉积. 最近, 有人报道在近赤道 地区也有冷水沉积 ${ }^{[74]}$. 此外, 大冰期以后, 瓜德鲁普 世末和长兴期中晚期可能还存在短暂的气候变冷事 件, 其中瓜德鲁普世末的气候变冷事件可能是瓜德 鲁普世巨型生物的杀手 ${ }^{[77]}$.

\section{3 新元古代-寒武纪与二叠-三叠纪转折时期 的地球化学异常事件}

伴随新元古代-寒武纪与二叠-三叠纪转折时期 发生的一系列生物与地质事件, 地层中记录了多次 与大气圈、水圈和生物圈组成变化密切相关的包括 $\mathrm{C}, \mathrm{O}, \mathrm{S}$ 和 $\mathrm{Sr}$ 等同位素地球化学的大幅度波动.

世界各地的许多剖面研究表明碳同位素 $\left(\delta^{13} \mathrm{C}\right)$ 在 雪球事件以后( 6.3 亿年)、埃迪卡拉纪中期 $(\sim 5.8$ 亿 年)、埃迪卡拉纪晚期( 5.6 亿年)以及新元古代与寒武 纪之交分别发生 4 次负漂移, 波动幅度达到 6\% 16\%o(图 1，2), 并在随后的寒武纪生物大爆发前 夕, 碳同位素进入一个强烈的波动期 ${ }^{[11,24,31,78]}$. 这 4 次碳同位素负漂移均伴随有紧随其后的生物辐射事 件 ${ }^{[24,26,79]}$, 早寒武世的碳同位素的强烈波动可能与随
后的生物大辐射(寒武纪大懪发)有密切相关性. 与海 水碳同位素变化相对应, 新元古代-寒武纪早期海水 中溶解硫酸盐的 $\mathrm{S}$ 同位素和地层中硫化物含量的变化 也非常大, 可以反映出当时古海洋生物地球化学循 环以及当时海水氧化还原条件的巨大变化. 最近的 研究表明在雪球事件以前古海水硫酸盐的 $\delta^{34} \mathrm{~S}$ 值呈 逐渐降低的趋势, 雪球事件以后 $\mathrm{S}$ 同位素发生大幅度 的变化, $\delta^{34} \mathrm{~S}$ 值迅速增大, 在短期内出现超过 $20 \%$ 的 正漂移, 达到 $40 \%$ 50\% $0^{[80,81]}$, 然后逐渐降低; 在新 元古代末有一次明显的增加 ${ }^{[82]}$, 寒武纪最早期再次 降到 $16 \%$ 左右, 随后, 迅速恢复到正常的 $18 \%$ 23\% ${ }^{[83]}$. 这种古海水的C, S 同位素组成大幅度 的变化和相伴随的地质和生物事件存在着内部联系, 说明不仅仅当时大气中的氧气含量, 而且海水中硫 酸盐和硫化氢含量变化也可能是控制生物辐射的主 要因素 ${ }^{[10,84]} . \mathrm{Sr}$ 同位素分析表明在整个新元古代是一 个持续缓慢增加的阶段 ${ }^{[85]}$, 在新元古代与寒武纪之 交之下有一次明显的快速正漂移, 比 $\delta^{13} \mathrm{C}$ 同位素的负 异常层位略低, 表明冈瓦纳大陆重组可能对当时陆 表环境变化产生重大影响 ${ }^{[86]}$. 除了上述地层记录的 同位素变化以外, $\mathrm{Mn} / \mathrm{Sr}, \mathrm{Th} / \mathrm{U}$ 和 $\mathrm{V} / \mathrm{Sc}$ 等的比值变化 反映出来海水介质也发生了明显的变化 ${ }^{[18,87]}$, 同时 伴随有缺氧事件和贯穿整个新元古代-寒武纪转折时 期的磷酸盐和黑色页岩沉积等. 所有上述地球化学 异常现象都表明新元古代-寒武纪转折时期是地球岩 石圈、生物圈、大气圈和水圈均处于一个剧烈变化的 关键时期.

石炭纪晚期-中三叠世的地层同样记录了与生物 和地质事件相伴生的 $\mathrm{C}, \mathrm{O}, \mathrm{Sr}$ 和 $\mathrm{S}$ 等同位素的异常变 化(图 1,2). 早石炭世有一次 $\delta^{13} \mathrm{C}$ 值快速正漂的过程, 可能与大陆冰川开始发育有关. 从晚石炭世至瓜德 鲁普世末期, $\delta^{13} \mathrm{C}$ 值在 $2 \%$ 4\%o 之间的波动 ${ }^{[12]}$ (图 1). 虽然有多次与冰期和间冰期转换有关的碳同位素波 动, 但幅度均较小. 值得一提的是乌拉尔世从 Asselian至Kungurian早期总体上 $\delta^{13} \mathrm{C}$ 值是一个逐渐 缓慢降低的过程 ${ }^{[8]}$, 在Sakmarian与Artinskian之间有 一次短暂的恢复, 说明当时气候逐渐由冰室效应期 转向温室效应期. 根据日本的剖面研究, 瓜德鲁普世 晚期有一次小规模的正漂移事件, 后者可能反映了 一次小规模的气候变冷事件 (Kamura事件) $)^{[77]}$. 伴随 着瓜德鲁普世末的生物灭绝事件, $\delta^{13} \mathrm{C}$ 有一次小幅降 低 ${ }^{[89,90]}$. 乐平世早期 $\delta^{13} \mathrm{C}$ 值为 $4 \%$ 。左右, 从吴家坪期 
中期开始直至早三叠世末期 $\delta^{13} \mathrm{C}$ 进入一个强烈的波 动期 ${ }^{[13,14]}$ (图 1,2). 伴随着二叠纪末生物大灭绝的突 然发生, $\delta^{13} \mathrm{C}$ 在 30 万年以内有一次约 5\% 7\%o的快速 负漂移, 这次负漂移在全世界范围内几乎所有的剖 面都有反映 ${ }^{[91]} . \delta^{34} \mathrm{~S}$ 和 ${ }^{87} \mathrm{Sr} /{ }^{86} \mathrm{Sr}$ 在瓜德鲁普世末和二 叠-三叠纪之交都有非常明显的负异常 ${ }^{[13,92,93]}$. 二叠 纪末生物大灭绝以后超长的生物萧条期(约 5 百万年) 和碳同位素的强烈震荡时期还伴随有大规模的缺氧 事件 ${ }^{[56,94,95]}$.

新元古代-寒武纪和二叠-三叠纪转折时期大规 模的 $\mathrm{C}, \mathrm{O}, \mathrm{Sr}$ 和 $\mathrm{S}$ 等同位素的异常变化等非常清晰地 表明当时发生了多次与地球水圈、大气圈地球化学组 成相关的变化 ${ }^{[24,82,86,96]}$, 包括氧气含量 ${ }^{[79,97,98]}$ 和大气 $\mathrm{CO}_{2}$ 的变化 ${ }^{[61]}$ 、海水介质条件的变化 ${ }^{[18]}$ 等. 此外, 雪 球事件以后和二叠纪中、晚期全球范围内大量发育的 碳酸盐岩沉积也有成对出现的现象. Knoll等 ${ }^{[10]}$ 和 Grotzinger和 Knoll ${ }^{[20]}$ 认为新元古代晚期和二叠纪晚 期海水是分层的, 深层缺氧并富含碱性碳酸盐的海 水由于上升流的作用或其他机制上升与浅层海水相 混, 从而导致大量文石和方解石沉淀, 形成大规模的 碳酸盐岩沉积. 雪球事件以后的碳酸盐岩盖帽和二 叠纪瓜德鲁普世在美国Texas和华南地区大规模发育 的生物礁和英国的富镁碳酸盐岩等都可能是在这种 机制下形成的 ${ }^{[20]}$.

\section{4 分析与对比}

上述两个关键时段生物多样性、地质事件背景和 海洋地球化学变化特征的比较给了我们诸多的启示. 一方面, 两个时间段重复发生了一系列重大地质事 件, 包括古大陆重组、大规模冰期等, 与这些事件相 伴生的是古海洋地球化学条件的改变和地表环境的 巨变, 环境的改变导致一系列重大生物灭绝和辐射 事件的发生; 另一方面, 这一系列事件的具体型式和 过程又因时而异.

\section{1 地球深部活动可能是地表一系列重大环境事 件的起因}

地球深部岩浆活动导致的地表板块运动、大陆重 组以及大量地幔物质释放到地表或大气对整个地球 系统所带来的影响是两个关键时段最具有科学意义 的共同特征, 也是地表环境发生重大变化, 并导致生
物界重大变革的主要动因. 地球深部活动导致的大 火成岩省一方面直接释放出大量的 $\mathrm{CO}_{2}, \mathrm{SO}_{2}, \mathrm{Cl}$ 和 $\mathrm{F}$ 等气体, 从而导致酸雨、温室效应、加快大陆风化作 用等; 另一方面火山活动还会引发海底沉积物不稳 定或对大陆地表富有机质沉积物加热导致甲烷气体 大规模释放, 从而造成地球表层的剧烈环境效 应 $^{[2,99 \sim 101]}$. 前寒武纪的Rodinia大陆的裂解和随后的 冈瓦纳大陆重组伴随着整个新元古代至早寒武世这 一演化阶段, 并伴随有与地幔柱活动相关的大火成 岩省出现 ${ }^{[32,102 ~ 104]}$. 地球深部活动对地球的影响还表 现在地球磁场的快速反复变化 ${ }^{[105]}$, 尽管新元古代和 寒武纪早期的地球磁极性变化还有待于研究, 从二 叠-三叠纪转折时期的变化(图 1)推测新元古代-寒武 纪之交可能也存在频繁的变化 ${ }^{[106]}$, 并已经得到初步 证实 ${ }^{[107]}$. 雪球事件之后盖帽白云岩中 $\delta^{13} \mathrm{C}_{\mathrm{carb}}$ 一度降 低到-48\% , 表明大规模甲烷突然释放事件的存在 ${ }^{[108]}$. 华南地区峨眉山大火成岩省、乐平统中几十层火山灰 层沉积和西伯利亚大火成岩省的存在充分说明了由 地球深部活动导致的火山活动频繁发生. 二叠纪末 期伴随生物大灭绝在短于 30 万年的时间内造成碳同 位素 5\%o 7\%o的负漂移, 除了大规模火山喷发的原因 以外, 甲烷释放也难逃其责, 并可能起到主导作用.

尽管上述两个时段均发生了联合大陆的聚散过 程，但所发生的时间、程度和持续时间均有明显不同. 前寒武纪的 Rodinia 大陆的裂解开始于雪球事件以前, 而石炭-二叠纪 Pangea 大陆的分裂是在石炭纪大冰期 之后, 而整个冰期过程是 Pangea 联合古陆的聚合时 期(图 1).

\section{2 大规模冰期是生物多样性增长的前奏}

从两个阶段的比较和整个地质历史记录来看, 大规模冰期是地球生物多样性相对偏低的时期, 但 随后向温室效应转变的过渡时期往往是生物多样性 的增长期. 发生于新元古代的雪球事件揭开了紧随 其后的多细胞动物辐射的序幕 ${ }^{[65]}$, 雪球事件以后, 在 埃迪卡拉纪早期宏观多细胞藻类和大型带刺疑源类 在海洋中首先开始大量出现. 而大型复杂的埃迪卡 拉生物辐射则发生在 5.8 亿年前的一次小规模的 Gaskier冰期事件之后 ${ }^{[32]}$ (图 2). 奥陶纪末的冰川事件 曾造成大量Ashgill期繁盛的动物群灭绝和随后凉水 型的Hirnantia动物群的广泛发育 ${ }^{[109]}$, 生物多样性明 显下降, 紧随其后的是生物多样性的一个快速增长 
期 $^{[42,110]}$. 与雪球事件时期不同, 石炭纪-早二叠世的 大冰期发生在Pangea大陆的聚合时期(图 1), 其冰雪 覆盖规模可能远比不上新元古代的雪球事件, 当时 陆地上已经形成最大规模的森林, 是显生宙最大规 模的碳埋藏时期, 大气中 $\mathrm{O}_{2}$ 含量高, $\mathrm{CO}_{2}$ 含量低, 与 陆地生态系统还没有形成的新元古代有很大不同, 因此, 雪球事件和石炭-二叠纪大冰期的生物、地质背 景和形成机制可能完全不同. 最新的研究表明石炭 纪-乌拉尔世是地球生物相对稳定发展的时期 ${ }^{[45,111]}$, 但生物多样性相对较低, 大冰期结束以后生物多样 性在二叠纪中、晚期呈快速增长 ${ }^{[110]}$. 第四纪冰期可 能对生物多样性的降低也有显著影响. 说明大冰期 及其以后与温室效应的过渡期对生物多样性的变化 有重要影响.

\section{3 新元古代与寒武纪转折时期发生了多次重大 生物更替事件}

由于化石数量有限, 新元古代-寒武纪转折时期 的生物更替无法从量化的角度与二叠纪末的生物大 灭绝进行比较, 但它所带来的生物变革的实际意义 可能超过显生宙的任何一次生物大灭绝. 在新元古 代与寒武纪之交整个埃迪卡拉生物群灭绝, 生物骨 骼化、生物行为的复杂化、生态空间的扩张在寒武纪 早期快速发生. 虽然大量胚胎化石和一批分类位置 不明的生物在新元古代晚期已经存在, 但大多数现 生动物门类的化石至今在埃迪卡拉纪还没有可靠的 报道. 这种新元古代与寒武纪之交生物的革命性更 替与二叠纪末生物大灭绝以后的中三叠世生物辐射 基本上是古生代生物门类的延续是一种不同生态层 次上的生物事件. 从梅树村小壳生物群的大量出现 到标志现生动物门类的首次大规模出现的澄江生物 大爆发经历了约 21 百万年 ${ }^{[24]}$, 新元古代至寒武纪早 期为许多动物门类的起源和辐射时期, 是地球生态 系统的建立阶段, 生物产生大量新的演化新质, 生物 的差异度快速增长(图 2), 原始的大量空白生态空间 需要去利用. 而以大量单调的双壳类和菊石类为主 的整个早三叠世生物复苏期经历的时间为 5 百万 年 ${ }^{[16,55]}$, 尽管远远长于显生宙历次生物大灭绝后的 复苏, 但仅仅是寒武纪初期小壳动物群所经历时间 的 1/4. 与新元古代-寒武纪转折时期所展示的崭新的 生态系统建立不同 ${ }^{[52]}$, 二叠纪末虽然造成大量生物 灭绝、生物礁消失和成煤作用停止, 生物灭绝主要发
生在纲、目一级水平, 三叠纪初分异度和差异度均明 显降低(图 2), 但古生代繁盛的各主要门类仍然延续, 很少有机会产生新的生物造型, 是一种生物界结构 和组成的重大变化. 大灭绝后, 只要环境合适, 残存 的生物类群就可以很快重新占领生态空间. 因此, 早 三叠世末期开始的中生代生物大辐射基本上是古生 代生物门类的延续. 早三叠世复苏期间的生物门类 数量也远远多于寒武纪早期(图 2), 其变化主要发生 在二级生态级别上, 要低于新元古代-寒武纪转折时 期一级生态系统的新生 ${ }^{[52]}$, 这表明新元古代与寒武 纪转折时期生物的生态类型转变强度要超过二叠-三 叠纪转折时期生物群的变更(图 2).

\section{4 地表环境剧变是生物大灭绝的主因}

从新元古代末-寒武纪和二叠-三叠纪两个关键 转折时期生物多样性变化与多种同位素地球化学指 标的变化规律对照来看, 两个转折时期同处于一个 碳同位素剧烈波动的时期, 从而表明生物发生大灭 绝与当时地表海洋和大气环境的剧烈变化具有时间 上的对应关系. 雪球事件以后的生物多次辐射和消 亡与当时海洋的氧化还原条件和大气氧气含量的变 化有密切关系 ${ }^{[79,84]}$. 新元古代晚期大型带刺疑源类 的灭绝、埃迪卡拉生物群在新元古代与寒武纪之交的 大规模灭绝以及发生在二叠纪末期的生物大灭绝均 伴随大幅度的 C, Sr和 S等同位素的异常现象(图 1, 2), 其中碳同位素大幅度快速负异常是一个最值得关注 的现象之一.

尽管新元古代末与寒武纪和二叠纪与三叠纪两 个转折时期均伴随有大规模地球化学指标的频繁波 动, 两者的波动幅度和波动范围有很大不同(图 2). 新元古代-寒武纪转折时期的 $\delta^{13} \mathrm{C}_{\mathrm{carb}}$ 在 $0 \%$ o左右波动, 其波动的幅度大, 可以从 $-10 \%$ 以下到 $6 \%$, 说明当 时整个生态系统还处于很不稳定的状态, 新元古界 与寒武系界线附近主要在-6\% 6\% 之间, 整个波动 期的时间较长, 频率相对较低, 大约每 4 百万年一个 周期(包括一次正漂移和一次负漂移); 而二叠-三叠 纪的 $\delta^{13} \mathrm{C}_{\mathrm{carb}}$ 平均在 $2 \%$ o左右波动, 其波动范围较小, 从- $4 \%$ o到 $6 \%$ 。左右, 整个波动期时间较短, 频率较高, 平均大约每 1 百万年一个周期(图 2). 从而说明新元古 代-寒武纪与二叠-三叠纪转折时期的大气 $\mathrm{CO}_{2}, \mathrm{O}_{2}$ 和 海水介质条件存在较大差距. 新元古代-寒武纪和二 叠-三叠纪转折期碳同位素异常与生物灭绝和辐射密 
切相关，正异常时期是生物繁盛或辐射期，而生物大 灭绝与负漂移相伴随 ${ }^{[11,73]}$ (图 1). 但是, 并不是所有 的碳同位素异常都伴随有生物大灭绝事件的发 生 ${ }^{[12,112]}$, 除了异常变化以外, 还取决于变化的幅度 和速度, 只有当环境变化超过了古生态系统的忍耐 度, 生物事件才会发生. 这可能也是两个关键时段一 系列生物事件在表现型式有所不同的原因.

\section{5 结语}

联合古陆、大规模的冰期、 $\mathrm{C}, \mathrm{Sr}$ 和 $\mathrm{S}$ 等同位素 的强烈波动以及生物多次大灭绝和辐射等发生在新 元古代-寒武纪与二叠-三叠纪转折时期的重大事件 重复出现给我们带来了新思考. 联合大陆的形成与
裂解以及伴随的火山活动、大规模气候(如冰期)和环 境变化事件成为地球生物巨变的动因, 与大幅度同 位素的快速漂移和环境巨变相互相成, 但生物演化 的进程和速度又因时而异. 通过对这两个关键时段 所发生的各类地质事件的比较将为研究地质历史时 期生物的更替、生物与环境的协同演化过程以及评估 当今地球的环境提供重要依据. 地球深部活动与地 表生物的响应是一个非常复杂的系统过程, 尽管目前 具体的原因还众说纷纭, 但可以肯定, 地质历史中在 某些关键时段一系列重大地质与生物事件相继发生绝 不是一个偶然的现象. 生物演化的机制要从全球的角 度着手, 从地球系统整体观出发, 把握科学前沿, 开 展从地球深部到地表过程的交叉研究对认识地球系统 将起到重要作用或产生重大影响的科学问题.

致谢 Matthew Clapham 和 Jonathon Payne 博士提供了二叠-三叠纪全球生物差异度和分异度数据，两位匿名审稿人 提出了许多宝贵的修改意见，在此表示衰心感谢.

\section{参考文献}

1 汪品先. 穿丵地球系统的时间隧道. 中国科学 D 辑: 地球科学, 2009, 39: 1313-1338

2 汪品先. 地球深部与表层的相互作用. 地球科学进展, 2009, 24: 1331-1338

3 Anbar A D, Knoll A H. Proterozoic ocean chemistry and evolution: A bioinorganic bridge? Science, 2002, 297: 1137-1142

4 Hallam A, Wignall P B. Mass extinctions and sea-level changes. Earth-Sci Rev, 1999, 48: 217—250

5 Erwin D H. Extinction: How Life on Earth Nearly Ended 250 Million Years Ago. New Jersey: Princeton University Press, 2006. 296

6 McGhee G R. The Late Devonian Mass Extinction. New York: Columbia University Press, 1996. 303

7 Zhou M F, Malpas J, Song X Y, et al. A temporal link between the Emeishan large igneous province (SW China) and the end-Guadalupian mass extinction. Earth Planet Sci Lett, 2002, 196: 113-122

8 Sepkoski J J Jr. A factor analytic description of the Phanerozoic marine record. Paleobiology, 1981, 7: 35-53

9 Bambach R K. Phanerozoic biodiversity mass extinctions. Annu Rev Earth Planet Sci, 2006, 34: 127—155

10 Knoll A H, Bambach R K, Canfield D E, et al. Comparative Earth history and Late Permian mass extinction. Science, 1996, 273: 452-457

11 Zhu M Y, Zhang J M, Yang A H. Integrated Ediacaran (Sinian) chronostratigraphy of South China. Palaeogeogr Palaeoclim Palaeoecol, 2007, 254: 7-61

12 Grossman E L, Yancey T E, Jones T E, et al. Glaciation, aridification, and carbon sequestration in the Permo-Carboniferous: The isotopic record from low latitudes. Palaeogeogr Palaeoclim Palaeoecol, 2008, 268: 222-233

13 Shen S Z, Henderson C M, Bowring S A, et al. High-resolution Lopingian (Late Permian) timescale of South China. Geol J, 2010, 45: 122 $-134$

14 Payne J L, Lehrmann D J, Wei J Y, et al. Large perturbations of the carbon cycle during recovery from the end-Permian extinction. Science, 2004, 305: 506-509

15 Gradstein F M, Ogg J G, Smith A G. A Geologic Time Scale 2004. Cambridge: Cambridge University Press, 2004. 589

16 Mundil R, Palfy J, Renne P R, et al. The Triassic timescale: New constraints and a review of geochronological data. Geol Soc London Spec Publ, 2010, 334: 41-60

17 Erwin D H. Biospheric perturbations during Gondwanan times: From the Neoproterozoic-Cambrian radiation to the end-Permian crisis. J Afr Earth Sci, 1999, 28: 115-127 
18 Kimura H, Watanabe Y. Oceanic anoxia at the Precambrian-Cambrian boundary. Geology, 2001, 29: 995-998

19 Erwin D H, Valentine J W, Sepkoski J J Jr. A comparative study of diversification events—The Early Paleozoic versus the Mesozoic. Evolution, 1987, 41: 1177-1186

20 Grotzinger J P, Knoll A H. Anomalous carbonate precipitates: Is the precambrian the key to the Permian? Palaios, 1995 , 10: 578-596

21 Marshall C R. Explaining the Cambrian “explosion” of animals. Annu Rev Earth Planet Sci, 2006, 34: 355-384

22 Knoll A H, Carroll S B. Early animal evolution: Emerging views from comparative biology and geology. Science, 1999, 284: 2129-2137

23 陈均远. 动物世界的黎明. 南京: 江苏科学技术出版社, 2004. 366

24 Zhu M Y, Strauss H, Shields G A. From snowball Earth to the Cambrian bioradiation: Calibration of Ediacaran-Cambrian Earth history in South China. Palaeogeogr Palaeoclim Palaeoecol, 2007, 254: 1-6

25 Chen J Y, Bottjer D J, Davidson E H, et al. Phosphatized polar lobe-forming embryos from the Precambrian of Southwest China. Science, 2006, 312: $1644-1646$

26 Li G X, Steiner M, Zhu X J, et al. Early Cambrian metazoan fossil record of South China: Generic diversity and radiation patterns. Palaeogeogr Palaeoclim Palaeoecol, 2007, 254: 229-249

27 Shen B, Dong L, Xiao S H, et al. The Avalon explosion: Evolution of Ediacara morphospace. Science, 2008, 319: 81-84

28 Yuan X L, Xiao S H, Taylor T N. Lichen-like symbiosis 600 million years ago. Science, 2005, 308: 1017-1020

29 Fedonkin M A, Gehling J G, Grey K, et al. The Rise of Animals: Evolution and Diversification of the Kingdom Animalia. Baltimore: Johns Hopkins University Press, 2003. 343

30 Chen J Y. The sudden appearance of diverse animal body plans during the Cambrian explosion. Int J Dev Biol, 2009, 53: 733-751

31 Zhou C M, Xie G W, McFadden K, et al. The diversification and extinction of Doushantuo-Pertatataka acritarchs in South China: Causes and biostratigraphic significance. Geol J, 2007, 42: 229-262 Condon D, Zhu M Y, Bowring S A, et al. U-Pb ages from the Neoproterozoic Doushantuo Formation, China. Science, 2005, 308: 95-98 Braiser M D. The basal Cambrian transition and Cambrian bio-events (from terminal Proterozoic extinctions to Cambrian biomers). In: Walliser O H, ed. Global Events and Event Stratigraphy in the Phanerozoic. Berlin: Springer-Verlag, 1995. 113-118 Sokolov B S, Fedonkin M A. Global biological events in the late Precambrian. In: Walliser O H, ed. Global Bio-events: Lecture Notes in Earth Sciences 8. Berlin, Heidelberg, New York: Springer, 1986. 105-108

35 McMenamin M A S, McMenamin D L S. The Emergence of Animals: The Cambrian Breakthrough. New York: Columbia University Press, 1989. 217

36 Zhu M Y. Precambrian-Cambrian trace fossils from eastern Yunnan: Implications for Cambrian explosion. Bull Nat Mus Nat Sci, 1997, 10: $275-312$

37 Bottjer D J, Hagadorn J W, Dornbos S Q. The Cambrian substrate revolution. GSA Today, 2000, 10: 1—7

38 Valentine J W. Prelude to the Cambrian explosion. Annu Rev Earth Plenat Sci, 2002, 30: 285-306

39 Meert J G, Lieberman B S. The Neoproterozoic assembly of Gondwana and its relationship to the Ediacaran-Cambrian radiation. Gondwana Res, 2008, 14: 5-21

40 Shu D G. Cambrian explosion: Birth of tree of animals. Gondwana Res, 2008, 14: 219-240

41 Sepkoski J J Jr. A compendium of fossil marine animal families. Milwaukee Public Mus Contrib Biol Geol, 1992, 83: 1-156

42 Rong J Y, Fan J X, Miller A I, et al. Dynamic patterns of latest Proterozoic-Palaeozoic-Early Mesozoic marine biodiversity in South China. Geol J, 2007, 42: 431-454

43 Hallam A, Wignall P B. Mass Extinctions and Their Aftermath. Oxford: Oxford University Press, 1997. 320

44 Jin Y G. Pre-Lopingian benthos crisis. Comptes Rendus XII ICC-P 2, 1993, 2: 269-278

45 Shen S Z, Zhang H, Li W Z, et al. Brachiopod diversity patterns from Carboniferous to Triassic in South China. Geol J, 2006, 41: 345-361

46 Jin Y G, Zhang J, Shang Q H. Two phases of the end-Permian mass extinction. In: Embry A F, Beauchamp B, Glass D J, eds. Pangea: Global Environments and Resources. Can Soc Petrol Geol, 1994, 17: 813-822

47 Stanley S M, Yang X N. A double mass extinction at the end of the Paleozoic Era. Science, 1994, 266: 1340-1344

48 Shen S Z, Shi G R. Paleobiogeographical extinction patterns of Permian brachiopods in the Asian-western Pacific region. Paleobiology, 2002, 28: $449-463$

49 Wang X D, Sugiyama T. Diversity and extinction patterns of Permian coral faunas of China. Lethaia, 2000, 33: 285-294 
50 Shen S Z, Shi G R. Latest Guadalupian brachiopods from the Guadalupian/Lopingian boundary GSSP section at Penglaitan in Laibin, Guangxi, South China and implications for the timing of the pre-Lopingian crisis. Palaeoworld, 2009, 18: 152-161

51 Yang X N, Liu J R, Shi G J. Extinction process and patterns of Middle Permian fusulinaceans in southwest China. Lethaia, 2004, 37: 139147

52 Droser M L, Bottjer D J, Sheehan P M. Evaluating the ecological architecture of major events in the Phanerozoic history of marine invertebrate life. Geology, 1997, 25: 167-170

53 Jin Y G, Wang Y, Wang W, et al. Pattern of marine mass extinction near the Permian-Triassic boundary in South China. Science, 2000, 289: $432-436$

54 Retallack G J. Permian-Triassic life crisis on land. Science, 1995, 267: 77-80

55 Lehrmann D J, Ramezani J, Bowring S A, et al. Timing of recovery from the end-Permian extinction: Geochronologic and biostratigraphic constraints from south China. Geology, 2006, 34: 1053-1056

56 Grice K, Cao C Q, Love G D, et al. Photic zone euxinia during the Permian-Triassic superanoxic event. Science, 2005, 307: 706-709

57 Xie S C, Pancost R D, Yin H F, et al. Two episodes of microbial change coupled with Permo/Triassic faunal mass extinction. Nature, 2005, 434: $494-497$

58 Cao C Q, Love G D, Hays L E, et al. Biogeochemical evidence for euxinic oceans and ecological disturbance presaging the end-Permian mass extinction event. Earth Planet Sci Lett, 2009, 281: 188-201

59 吴亚生, 姜红霞, Yang W, 等. 二叠纪-三叠纪之交缺氧环境的微生物和微生物岩. 中国科学 D 辑: 地球科学, 2007, 37: 618一 628

60 Piper J D A. The Neoproterozoic supercontinent: Rodinia or palaeopangaea? Earth Planet Sci Lett, 2000, 176: 131-146

61 Hoffman P F. The break-up of Rodinia, birth of Gondwana, true polar wander and the snowball Earth. J Afr Earth Sci, 1999, 28: 17-33

62 Meert J G, Lieberman B S. A palaeomagnetic and paleobiogeographical perspective on latest Neoproterozoic and early Cambrian tectonic events. J Geol Soc London, 2004, 161: 477-487

63 Evans D A D. A fundamental Precambrian-Phanerozoic shift in Earth's glacial style? Tectonophysics, 2003, 375: 353-385

64 Zheng Y F, Wu Y B, Gong B, et al. Tectonic driving of Neoproterozoic glaciations: Evidence from extreme oxygen isotope signature of meteoric water in granite. Earth Planet Sci Lett, 2007, 256: 196-210

65 Boyle R A, Lenton T M, Williams H T P. Neoproterozoic 'snowball Earth' glaciations and the evolution of altruism. Geobiology, 2007, 5: $337-349$

66 Yin L M, Zhu M Y, Knoll A H, et al. Doushantuo embryos preserved inside diapause egg cysts. Nature, 2007, 446: 661-663

67 袁训来, 肖书海, 尹否明, 等. 陡山陀期生物群一一早期动物辐射前夕的生命. 合肥: 中国科学技术大学出版社, 2002.171

68 Eyles N, Eyles C H. Glacially-influenced deep-marine sedimentation of the late Precambrian Gaskiers Formation, Newfoundland, Canada. Sedimentology, 1989, 36: 601-620

69 Scotese C R, Langford R P. Pangea and the paleogeography of the Permian. In: Scholle P A, Peryt T M, Ulmer-Scholle D S, eds. The Permian of Northern Pangea, Volume 1: Paleogeography, Paleoclimates, Stratigraphy. Berlin: Springer-Verlag, $1995.3-19$

70 Ziegler A M, Hulver M L, Rowley D B. Permian world topography and climate. In: Martini I P, ed. Late Glacial and Postglacial Environmental Changes_-Quaternary, Carboniferous-Permian and Proterozoic. New York: Oxford University Press, $1997.111-146$

71 Renne P R, Zhang Z C, Richards M A, et al. Synchrony and causal relations between Permian-Triassic boundary crises and Siberian flood volcanism. Science, 1995, 269: 1413-1416

72 Bowring S A, Erwin D H, Jin Y G, et al. U/Pb zircon geochronology and tempo of the end-Permian mass extinction. Science, 1998, 280: $1039-1045$

73 Korte C, Pande P, Kalia P, et al. Massive volcanism at the Permian-Triassic boundary and its impact on the isotopic composition of the ocean and atmosphere. J Asian Earth Sci, 2010, 37: 293-311

74 Poulsen C J, Pollard D, Montanez I P, et al. Late Paleozoic tropical climate response to Gondwanan deglaciation. Geology, 2007, 35: 771774

75 Veevers J J. Gondwanaland from 650-500 Ma assembly through 320 Ma merger in Pangea to 185-100 Ma breakup: Supercontinental tectonics via stratigraphy and radiometric dating. Earth-Sci Rev, 2004, 68: 1- 132

76 Isbell J L, Lenaker P A, Askin R A, et al. Reevaluation of the timing and extent of late Paleozoic glaciation in Gondwana: Role of the Transantarctic Mountains. Geology, 2003, 31: 977-980 
77 Isozaki Y, Kawahata H, Minoshima K. The Capitanian (Permian) Kamura cooling event: The beginning of the Paleozoic-Mesozoic transition. Palaeoworld, 2007, 16: 16-30

78 Ishikawa T, Ueno Y, Komiya T, et al. Carbon isotope chemostratigraphy of a Precambrian/Cambrian boundary section in the Three Gorge area, South China: Prominent global-scale isotope excursions just before the Cambrian Explosion. Gondwana Res, 2008, 14: 193-208

79 McFadden K A, Huang J, Chu X L, et al. Pulsed oxidation and bioloical evolution in the Ediacaran Doushantuo Formation. Proc Natl Acad Sci USA, 2008, 105: 3197-3202

80 Chu X L, Zhang Q R, Zhang T G, et al. Sulfur and carbon isotopic variations in Neoproterozoic sedimentary rocks from southern China. Prog Nat Sci, 2003, 13: 875-880

81 张同钢, 储雪蕾, 冯连君, 等. 新元古代 “雪球事件” 事件对海水碳、硫同位素组成的影响. 地球学报, 2003, 26: 487一 493

82 Shen Y, Zhao R, Chu X L, et al. The carbon and sulfur isotope signatures in the Precambrian-Cambrian Transition series of the Yangtze Platform. Precambrian Res, 1998, 89: 77-86

83 Goldberg T, Strauss H, Guo Q J, et al. Late Neoproterozoic to early Cambrian sulphur cycle-An isotopic investigation of sedimentary rocks from the Yangtze Platform. Prog Nat Sci, 2003, 13: 946-950

84 Li C, Love G D, Lyons T W, et al. A stratified redox model for the Ediacaran Ocean. Science, 2010, 328: 80-83

85 Halverson G P, Dudas F O, Maloof A C, et al. Evolution of the ${ }^{87} \mathrm{Sr} /{ }^{86} \mathrm{Sr}$ composition of Neoproterozoic seawater. Palaeogeogr Palaeoclim Palaeoecol, 2007, 256: 103-129

86 Sawaki Y, Ohno T, Fukushi Y, et al. Sr isotope excursion across the Precambrian-Cambrian boundary in the Three Gorges area, South China. Gondwana Res, 2008, 14: 134-147

87 Shen Y. C-isotope variations and paleoceanographic changes during the late Neoproterozoic on the Yangtze Platform, China. Precambrian Res, 2002, 113: 121-133

88 Korte C, Jasper T, Kozur H W, et al. $\delta^{18} \mathrm{O}$ and $\delta^{13} \mathrm{C}$ of Permian brachiopods: A record of seawater evolution and continental glaciation. Palaeogeogr Palaeoclim Palaeoecol, 2005, 224: 333-351

89 Kaiho K, Chen Z Q, Ohashi T, et al. A negative carbon isotope anomaly associated with the earliest Lopingian (Late Permian) mass extinction. Palaeogeogr Palaeoclim Palaeoecol, 2005, 223: 172-180

90 Wang W, Cao C Q, Wang Y. The carbon isotope excursion on GSSP candidate section of Lopingian-Guadalupian boundary. Earth Planet Sci Lett, 2004, 220: 57-67

91 Korte C, Kozur H W. Carbon-isotope stratigraphy across the Permian-Triassic boundary: A review. J Asian Earth Sci, 2010, 39: 215-235

92 Korte C, Jasper T, Kozur H W, et al. ${ }^{87} \mathrm{Sr} /{ }^{86} \mathrm{Sr}$ record of Permian seawater. Palaeogeogr Palaeoclim Palaeoecol, 2006, 240: 89-107

93 Kaiho K, Chen Z Q, Kawahata H, et al. Close-up of the end-Permian mass extinction horizon recorded in the Meishan section, South China: Sedimentary, elemental, and biotic characterization and a negative shift of sulfate sulfur isotope ratio. Palaeogeogr Palaeoclim Palaeoecol, 2006, 239: 396-405

94 Wignall P B, Twitchett R J. Oceanic anoxia and the end Permian mass extinction. Science, 1996, 272: 1155-1158

95 Isozaki Y. Permo-Triassic boundary superanoxia and stratified superocean: Records from lost deep sea. Science, 1997, 276: 235-238

96 Shen Y, Schidlowski M, Chu X L. Biogeochemical approach to understanding phosphogenic events of the terminal Proterozoic to Cambrian. Palaeogeogr Palaeoclim Palaeoecol, 2000, 158: 99-108

97 Kump L R. The rise of atmospheric oxygen. Nature, 2008, 451: 277-278

98 Canfield D E, Poulton S W, Narbonne G M. Late-Neoproterozoic deep-ocean oxygenation and the rise of animal life. Science, 2007, 315: $92-95$

99 Neal C R, Coffin M F, Arndt N T, et al. Investigating large igneous province formation and associated paleoenvironmental events: A white paper for scientific drilling. Scient Drilling, 2008, 6: 4-18

100 Payne J L, Lehrmann D J, Follett D, et al. Erosional truncation of uppermost Permian shallow-marine carbonates and implications for Permian-Triassic boundary events. Geol Soc Am Bull, 2007, 119: 771—784

101 Santosh M. A synopsis of recent conceptual models on supercontinent tectonics in relation to mantle dynamics, life evolution and surface environment. J Geodyn, 2010, 50: 116-133

102 Ernst R E, Wingate M T D, Buchan K L, et al. Global record of 1600-700 Ma Large Igneous Provinces (LIPs): Implications for the reconstruction of the proposed Nuna (Columbia) and Rodinia supercontinents. Precambrian Res, 2008, 160: 159-178 
103 Wang X C, Li X H, Li W X, et al. Variable involvements of mantle plumes in the genesis of mid-Neoproterozoic basaltic rocks in South China: A review. Gondwana Res, 2009, 15: 381-395

104 Wang X C, Li X H, Li W X, et al. The Bikou basalts in the northwestern Yangtze Block, South China: Remnants of 820-810 Ma continental flood basalts? GSA Bull, 2008, 120: 1478-1492

105 Haag M, Heller F. Late Permian to Early Triassic magnetostratigraphy. Earth Planet Sci Lett, 1991, 107: 42-54

106 Kirschvink J L, Raub T D. A methane fuse for the Cambrian explosion: Carbon cycles and true polar wander. Compt Rend Geosci, 2003, 335: $65-78$

107 Mitchell R N, Evans D A D, Kilian T M. Rapid Early Cambrian rotation of Gondwana. Geology, 2010, 38: 755—758

108 Wang J S, Jiang G Q, Xiao S H, et al. Carbon isotope evidence for widespread methane seeps in the ca. 635 Ma Doushantuo cap carbonate in south China. Geology, 2008, 36: 347-350

109 Rong J Y, Harper D A T. A global synthesis of the latest Ordovician Hirnantian brachiopod faunas. Trans R Soc Edinb-Earth Sci, 1988, 79: $383-402$

110 Alroy J, Aberhan M, Bottjer D J, et al. Phanerozoic trends in the global diversity of marine invertebrates. Science, 2008, 321: 97-100

111 Wang X D, Wang X J, Zhang F, et al. Diversity patterns of Carboniferous and Permian rugose corals in South China. Geol J, 2006, 41: 329 $-343$

112 Veizer J, Ala D, Azmy K, et al. ${ }^{87} \mathrm{Sr} /{ }^{86} \mathrm{Sr}, \delta^{13} \mathrm{C}$ and $\delta^{18} \mathrm{O}$ evolution of Phanerozoic seawater. Chem Geol, 1999, 161: 59—88 